\title{
SCTP-MANET NEW EXTENSION OF SCTP PROTOCOL FOR THE OPTIMIZATION OF MANET PERFormanCES
}

\author{
SADOUNI Salheddine, ${ }^{1}$ Benslama Malek ${ }^{1}$ and Beylot André-Luc ${ }^{2}$ \\ ${ }^{1}$ Electromagnetism and telecommunication laboratory, University of Constantine \\ ${ }^{2}$ IRIT, Université de Toulouse
}

\begin{abstract}
Ad Hoc mobile networks are constituted of nodes that move freely without a centralized administration. These nodes contribute in the routing of data packets that are sent by a source. This happens when the latter is not capable of reaching its destination. On the other hand, their mobility causes recurrent breakdowns of the routing paths notably with sparse MANET. In order to optimize the performance of such networks, we suggest a new extension of protocols: Stream Control Transmission Protocols (SCTP) named SCTP-MANET. Their main function is therefore to improve the availability of the links in sparse MANET protocols. This could be achieved by a better integration of Multihoming. With this aim in mind, this new extension is based on a cross-layer interface between transport and routing layers as well as the use of specific messages.
\end{abstract}

\section{KEYWORDS}

SCTP, AODV, Cross layer interface, Multihoming, Throughput

\section{INTRODUCTION}

The main purpose behind establishing Ad Hoc mobile networks is to widen the notions of mobility. Since they may contain mobile platforms - in other words nodes - which are free to move without constraints. These networks represent an autonomous system, capable of organizing itself without any previous infrastructure.

Radio transmissions being limited, every node is likely to be involved in the routing process in order to forward the packets of a node that could not reach its destination. This involves the use of three types of protocols: proactive, reactive, and hybrid. Proactive protocols try to maintain the best paths available in order to reach every possible destination. The routes are saved even when not used. On the contrary, Reactive protocols create and maintain the routes according to the needs. This could be achieved by the launch of a global discovery procedure aiming at finding out a path between the source node, and the destination one. As far as hybrid protocols are concerned, a proactive technique is involved when recognizing the topology locally. However, the routes leading to more remote nodes are attained through a reactive technique [1]. In this article, we will focus on the reactive routing category, more precisely on Ad Hoc on Demand Vector Routing protocols.

DOI: $10.5121 /$ ijwmn.2016.8504 
However, the main disadvantage of MANET is the perpetual instability of its topology as well as the number of the nodes participating in the data routing. A sparse MANET undergoes more link interruptions between source and destination. The intermediary nodes that do not have at disposal alternative routes, will thus delete the data packets. This in return shows weak network performances [2]. For such reasons, we suggest in this article a new extension of SCTP protocol. We call it: SCTP-MANET. Its main function would be to define the best method of integrating Multihoming to MANET. This should be done while taking into account its characteristics. The previously mentioned concept will focus on the creation of multiple paths between source and destination node, and ensuring the transparent passage of a broken-down node to one that works. In order to validate the contribution of this new extension to network performances in this article, we will be based upon a comparative study of the average throughput between SCTP-MANET application and the standard SCTP version in different MANET topologies. This could be done by using Riverbed Modeler software which represents a new version of Openet Modeler.

In order to do this, section two (2) will be devoted to tackle AODV routing protocol and its link failure detection mechanism. Section three (3) will broach SCTP transport protocol and how it handles Multihoming. The Cross-layer interface and SCTP-MANET new extension will be presented in section four (4). As far as section five (5) is concerned, we will present the simulation results obtained by the simulator Riverbed Modeler for which we have suggested an SCTP setting and some of the extensions tackled in this paper. Finally, section 6 will allow us to conclude and highlight the perspectives of this work.

\section{AODV Routing Protocols}

Ad Hoc on demand distance vector routing belongs to the category of reactive protocols. The latter create and maintain the routes according to the needs. They use a broadcast mechanism in order to discover the valid routes [3]. Therefore, two operations are defined.

\subsection{Routes Discovery}

Two types of messages are used [4]

- RREQ (Route REQuest)

- RREP (Route REPly)

A node initiates a process of route discovery when it needs a route to a new destination, or a destination to which the route has become invalid. The sender broadcasts the RREQ message in the network to find a path. The message is first received by the closest neighboring nodes which will in return spread the message until reaching the destination node. Each intermediary node stores in its routing table the address of the node that transmitted the RREQ message. Therefore, the reverse path is established.

Once the message reaches the destination node (or a node familiar with a path leading to this one), it sends a RREP message to the source using the reverse path. RREP travels along the path which is followed by the route request in the opposite direction, and modifies the routing tables of the nodes through which it passes.

\subsection{Routes Maintenance}

To repare a broken-down route, AODV uses the message [4]

- Route ERRor (RERP) 
International Journal of Wireless \& Mobile Networks (IJWMN) Vol. 8, No. 5, October 2016

During this operation, this protocol predicts a mechanism that will repair the route locally and save routing message transmissions. In addition, it anticipates optimizations to repair a route locally.

\subsection{Link Failure Detection}

AODV protocol suggests two methods to detect links state: The transmission of the message (Hello) at regular intervals [5], and the awareness of the information coming directly from MAC sublayer [6].

\section{SCTP TRANSPORT Protocol}

SCTP is a transport layer protocol which was designed by Internet Engineering Task Force (IETF) in order to provide a reliable and secure transport of a variety of applications. One of these most important applications is network signaling [7]. For the design of SCTP, two major functionalities have been introduced, depending on the stream and Multihoming [8].

Stream refers to a sequence of messages within the same SCTP association (chunk). As far as Multihoming is concerned, SCTP defines it as being the capacity of a terminal point to contain multiple interfaces with different IP addresses. Consequently, in the case of a multi-homed network connection, numerous paths could be established for each source interface. SCTP chooses a primary address that will be used as a destination for every data transfer. Thus, the other addresses are considered to be alternatives [8]. In case of primary address breakdown, SCTP uses the alternative addresses to forward the lost data until the link is re-established with the former one.

\subsection{SCTP Association}

An association is established by the exchange of four messages: INIT, INIT-ACK, COOKIEECHO and COOKIE-ACK. To initiate an association, the sender emits an INIT message to the receiver which answers it in return with an INIT-ACK message containing configuration information to constitute a COOKIE. The receiver analyzes the information that the received COOKIE-ECHO packet contains, in order to ensure that it is the original sender of the COOKIE message. If the key is valid, the receiver resends a COOKIE-ACK to the sender and considers the association to be established [9].

\subsection{SCTP ADDIP Dynamic Address Addition}

SCTP ADDIP is an SCTP extension defined in RFC 5061. It allows a dynamic update of the association address list. It is known as Mobile SCTP (mSCTP) [10]. It permits to a mobile that moves away of its home network to obtain a new address. The latter is integrated automatically to the association, and used to pursue a transparent transfer of data.

ADDIP adds two new types of control messages: Address Configuration Change (ASCONF) and Address Configuration Acknowledgment (ASCONF-ACK). These messages enable the addition, the suppression or the modification of the Address on the whole of IP addresses of the two association extremities [10]. 


\subsection{SCTP Multiple-Route Method}

During an SCTP association, the protocol verifies the validity of each communication route between two nodes. The latter's destination transport address is declared unoccupied if not used during a pre-defined period higher than or equal to an HB interval (30 seconds by default). A HEARTBEAT message is associated to a source transport address and to a destination one. It answers with a HEARTBEAT-ACK message [11]. A source node increments ERROR COUNTER parameter associated to a transport destination address when it does not receive a retransmission timeout.

According to the value of error counter parametre, SCTP allows to detect a route's rupture (error counter passess the threshold Path.Max.Retrans) or an unreachable destination node (the sum of error counter associated to the different destination node addresses is higher than the value of Association.Max.Retrans). When the primary route is marked inactive, the sender switches automatically its data traffic to an active alternative route without closing the association. On the other hand, it will continue to send Heartbeat chunks regularly to the primary route in order to verify its state. When the primary route becomes active again, SCTP protocol uses it to send the traffic over [12].

\subsection{SCTP and AODV Limits}

The integration of Multihoming to sparse MANET increases their performances by switching the stream of information from a broken-down route to one that works. That is why the use of SCTP that takes in charge Multihoming, combined with AODV routing protocol seems to be the most appropriate. Except that two situations arise in the present case:

1. AODV is a Unipath protocol that constitutes merely one route on demand between source and destination [13]. Thus, when this route is broken down, the intermediary nodes suppress the data packets, because they do not have an alternative route.

2. In addition, if on the level of transport, SCTP protocol has an alternative route, AODV routing protocol will not be aware of the existence of a second route when the primary one breaks down. Consequently, the source node launches a new route discovery operation [14].

For such reasons, we ought to suggest SCTP extensions that will be combined in a new protocol SCTP-MANET. The principles of the latter will be tackled in the section to come.

\section{THE DESCRIPTION OF THE NEW SCTP-MANET PROTOCOL}

In order to optimize the performances of sparse MANET by using a quick recovery of the broken down routes, it is crucial to compensate for its previously described shortcomings. Therefore, SCTP-MANET extension would be based upon two main elements:

1. Cross layer interface.

2. The use of specific messages by adding additional parameters.

These enhancements will thus focus on not only the architecture, but also on the messages of the SCTP protocol itself. 


\subsection{Cross Layer Interface}

The current architecture of network protocols lies on a set of modules that are bound to provide a particular service in an autonomous way. Due to their hierarchical organization in a stack, each module finds it difficult to communicate with the other modules.

In this paper, we will use a transport and routing cross layer interface that is based on the model of the interaction with an intermediary entity type [15]. This cross layer interface performs its data sharing by a service of recovery, storage and data sending between the transport layer and the routing one [16]. The data concerned with the recovery and the sharing are: RREQ, RREP, and RRER of AODV routing protocol. As well as the messages establishing an association of SCTP transport protocol: INIT and INIT-ACK.

In the case of primary path breakdown, the standard SCTP version launches a route discovery procedure to look for a new route. Whereas the use of the information of transport layer 4 by the routing layer 3, SCTP-MANET sends directly the packets through a second path. A primary route recovery procedure will be launched thereafter.

In order to optimize network resources and avoid signaling bursts signal peaks that are relative to the creation of multiple paths through every network node, we will implant CLI cross layers at the level of source nodes (SCLI) (as shown in figure 1) and destination nodes (DCLI) only (as shown in figure 2)

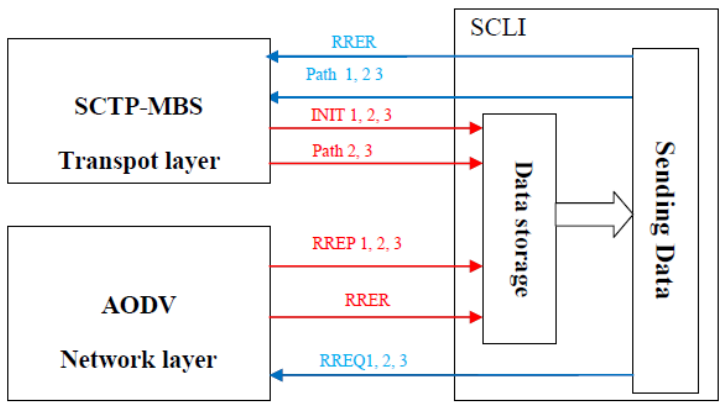

Figure 1. Cross Layer Interface Architecture - source node

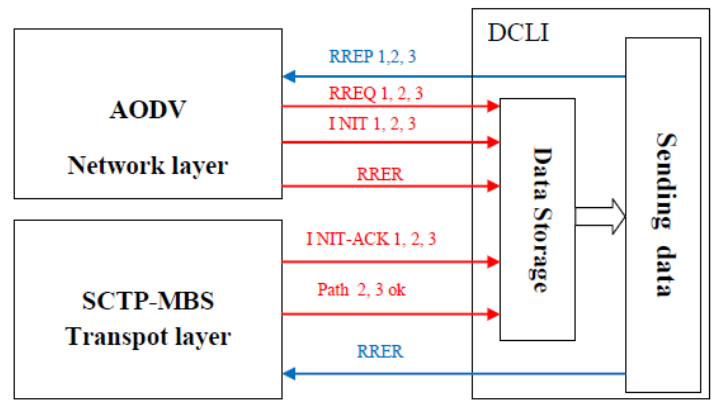

Figure 2. Cross Layer Interface Architecture - destination node 


\subsection{The Use of Specific Messages}

In addition to the use of a cross layer interface, SCTP-MANET, in order to function properly, is based upon specific messages by adding additional parameters. In the first place, INIT and INITACK messages are necessary for the initialization of an SCTP association. In the second place, RREQ and RREP routing messages are used by AODV protocol in route search and discovery.

SCTP-MANET starts by the creation of a primary route numbered 1 . Then two other secondary routes are numbered successively 2 and 3 . These numbers will be implemented using binary code in the flag field INIT and INIT-ACK. The eight flag bits are not used in control messages. Consequently, INIT 1 brings to the flag field the value 00000001; INIT 2 brings 00000010, and so on for the rest of the messages INIT3 and INIT-ACK from 1 to 3. (Figure 3)

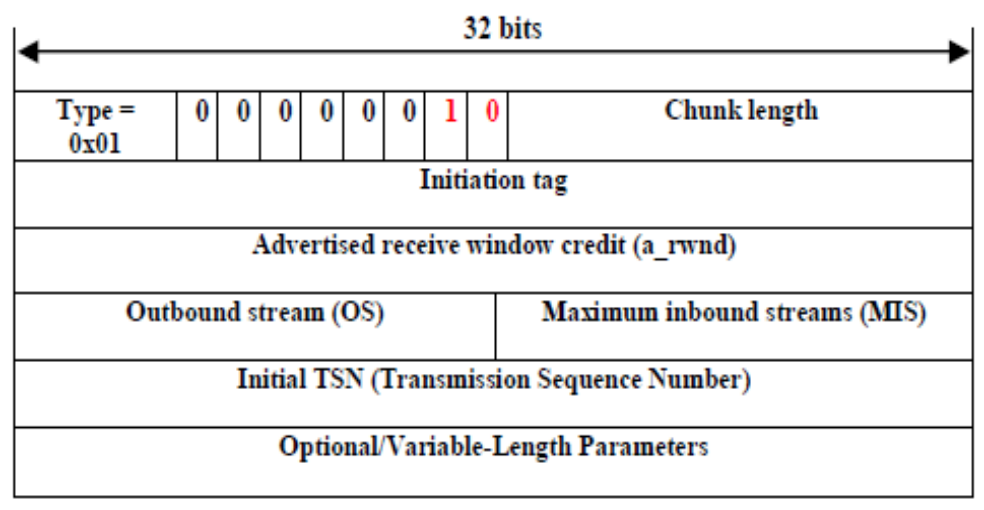

Figure 3. The Format of a Message INIT 2

As far as RREQ and RREP routing messages are concerned, their numbering is similar to that of INIT and INIT-ACK messages; from 1 to 3 . However, they are to be implemented in the fields RESERVED. Constituted of 9 bits to 0 for RREP and from 11 bits to 0 for RREQ successively, these fields are ignored once received [17]. Their numbering is binary coded. RREQ 1 will have the value 00000000001 and RREP 1000000001 and so on for the rest of RREQ and RREP messages.

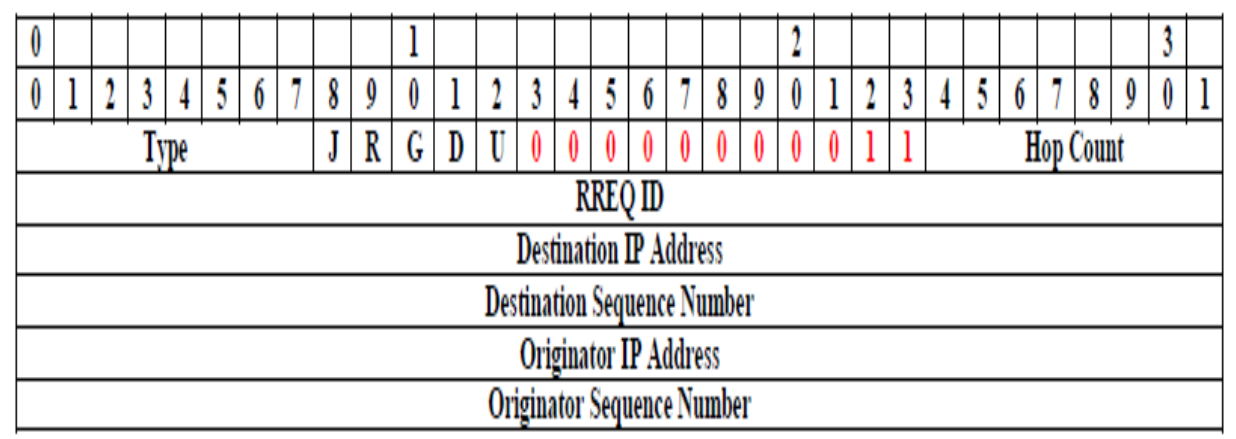

Figure 4. The Format of a Message RREQ 3

SCTP-MANET has been designed to operate simultaneously with three active paths: a direct route, and two rescue ones in case the first one breaks down. It is possible to schedule this 
extension to ceaselessly look for alternative paths. However, such a move could lead to routing signaling peaks which, in return, could result in saturating the network.

Along the same lines, the numbering of routing messages involves the sequential creation of routes in order of importance (primary route, the first alternative route, then finally the second alternative one). Particularly when knowing that AODV routing protocol uses a flooding-based route discovery [18].

Finally, a node which is requested by several routes simultaneously involves their interruption in case it moves. That is why disjointed routes should be created. In order to do this, a numbered RREP marks its number in the routing table of every node when passing to a destination node. Afterwards, once a new route discovery operation is launched, the numbered RREQ ignores the marked nodes with a number different than its own.

\subsection{SCTP-MANET Association Establishment Steps}

The objective of an SCTP-MANET association is to continuously operate with three paths (one primary path, and two secondary ones). In case of a primary path breakdown, SCTP-MANET automatically switches the data sending to the first secondary path without any disconnection if it is available. If not, it switches to a second alternative path. In order to do so, it achieves the following operations:

1. Over a first phase, the source node attempts to initialize a transport SCTP-MANET association. That is why it sends a message INIT1 to SCLI interface. The latter stores the INIT1 message, and asks AODV protocol to create a path to the destination node.

2. Therefore, AODV sends a RREQ1 using a diffusion based process. Once it reaches the destination node, DCLI stores it. Then, it answers with a RREP1.

3. On the way back, RREP1performs the same tasks as an ordinary RREP [4] (it changes the intermediary node routing tables). Except that this RREP also marks the nodes with its number as it passes.

4. Once RREP1 reaches the source node, SCLI sends INIT1 to the path that is previously established by AODV. The destination node resends an INIT-ACK1

5. Then, the SCTP association establishment procedure is initialized between source and destination node with the four messages: INIT1, INIT-ACK1, COOKIE-ECHO and COOKIE-ACK. The last two messages are not numbered.

6. After establishing an association between source and destination node, SCTP-MANET considers this path to be primary and relies on it to send the data packets.

7. Meanwhile, SCTP-MANET initializes the same route discovery operation with numbered 2 INIT, then with numbered 3 INIT.

8. That is why SCLI and DCLI take over and accomplish the same previously described steps. Except that a numbered RREQ ignores the marked nodes with another number different of its own one.

9. The return of a numbered RREP means the discovery of a secondary path to the destination. The corresponding message INIT-ACK carries the IP addresses participating in the association, and ADDIP takes over to include this new path.

10. The message COOKIE-ECHO will not be sent because the association already exists [19].

11. SCTP-MANET uses an active route surveillance mechanism thereafter, by sending HEARTBEAT chunks [11]. 
Eventually, when the primary route breaks down, SCTP-MANET seeks re-establishing it with the sending of RREQ1, but only after ten (10) RREQ1 sending attempts fail. Therefore SCTPMANET eliminates this route, and realizes a gap in its active route list.

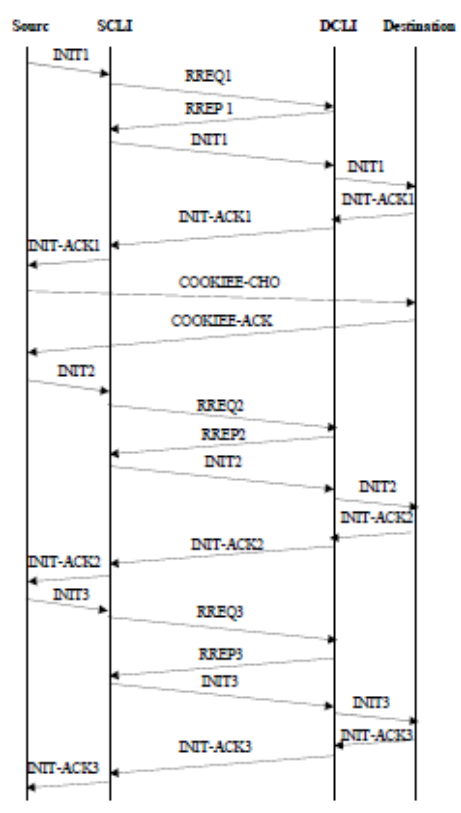

Figure 5. Source and Destination Communication with SCTP-MANET

\section{Simulation}

\subsection{Simulation Framework}

Riverbed Modeler Academic Edition 17.5 is a highly sophisticated simulation software package that enables developers to model communications networks and distributed systems and allows them to analyse the behaviour and performance of modelled systems through Discrete Event Simulations (DES) [20] This framework is the new version of Opnet Modeler network simulator, from which we could import network models, as well as scenarios to be executed in it. This software is one of the most popular, accurate and applicable in the real world in the field of network simulation and is recognized for its high reliability. It allows for the simulation of different scenarios for a specific project and uses a project and scenario approach to modeling networks. The project approaches a collection of related network scenarios in which each explores a different aspect of network design. It contains at least one scenario, that is, a single instance of a network. Simulating a scenario can overcome constraints of proprietary hardware and software such as lack of development tools. The Riverbed Modeler offers to its user a Graphic User Interface (GUI), standards-based LAN and WAN performance modeling and detailed library model for most protocols and devices [21].

\subsection{Performance Evaluation}

The collected statistic for this comparative study is Throughput, in communication of various types of networks, such as MANET, network throughput is the average rate of successful 
message delivery over a communication channel. The throughput is usually measured in bits per second (bit/s or bps) or in data packets per second or data packets per time slot. These data may be delivered over a physical or logical link, or pass through a certain network node. The system throughput or aggregate throughput is the sum of the data rates that are delivered to all terminals in a network. [22] Throughput analysis is one of several important parameter of network performance. A weak throughput implies that the transmission medium is scarcely requested by stations, or that the medium capacity is badly used by the access technique.

\subsection{Simulation Parameters}

In order to validate a qualitative contribution of our SCTP-MANET extension, we considered three different scenarios. Each one of them contains two variants:

1. The use of the standard version of SCTP protocol.

2. The use of the new extension SCTP-MANET.

Regarding the first variant, AODV protocol is activated in the third routing layer. The former already exists in the simulation framework. This applies to all the network nodes.

On the other hand, SCTP transport layer was not implanted in the simulation tool. We have set it up in its basic version. Same goes to the previously suggested extensions. In addition, it is noteworthy that this important work includes modifications of the automatons generally used in the transport layer. Particularly by adding relative states of COOKIE-ECHO and COOKIE-ACK message sending. These modifications apply only to the source and destination nodes.

Regarding the second variant, SCTP-MANET extension is designed to operate in the fourth layer. In order to do so, a Find-new state is added to the functioning of SCTP. The role of the latter will be to define the second and the third paths after SCTP-MANET association has been activated.

As far as cross-layer interfaces are concerned, two new processors are created: SCLI for the source node and DCLI for the destination node. They are situated between the layers three and four and joint by packets stream. These modifications only apply to source and destination nodes.

Finally, regarding the routing, AODV protocol is activated in all the network nodes. Numbered RREP and RREQ routing messages are taken into charge by SCLI and DCLI processors.

Table 1. Simulation model parameters used for SCTP-MANET

\begin{tabular}{|l|l|}
\hline Receive buffer (bytes) & 65535 \\
\hline Send buffer size & unlimited \\
\hline $\begin{array}{l}\text { Maximum (INIT1, COOKIE) ACK delay (sec) } \\
\text { for SCTP-MANET }\end{array}$ & 0.2 \\
\hline $\begin{array}{l}\text { Maximum (INIT2, INIT 3) ACK delay (sec) for } \\
\text { SCTP-MANET }\end{array}$ & 0.4 \\
\hline $\begin{array}{l}\text { Maximum (INIT, COOKIE) ACK delay (sec) for } \\
\text { SCTP }\end{array}$ & 0.2 \\
\hline Maximum ACK Segments & 2 \\
\hline Duplicate ACK Threshold & 3 \\
\hline Initial RTO (sec) & 1 \\
\hline
\end{tabular}


International Journal of Wireless \& Mobile Networks (IJWMN) Vol. 8, No. 5, October 2016

\begin{tabular}{|l|l|}
\hline Minimum RTO $(\mathrm{sec})$ & 0.5 \\
\hline Maximum RTO $(\mathrm{sec})$ & 64 \\
\hline
\end{tabular}

\subsection{Simulation Scenarios:}

In a MANET, every node is likely to contribute in the data routing. Thus, the number of the nodes that constitute a MANET influences directly the network performances. In order to observe this, we realize three scenarios:

1. The first scenario: a dense network of thirty (30) nodes.

2. The second scenario: a medium-density network of twenty (20) nodes.

3. The third scenario: a sparse network of ten (10) nodes.

Table 2. Simulation model parameters used for the three scenarios

\begin{tabular}{|l|l|}
\hline Data rate Mbps & 1 \\
\hline $\begin{array}{l}\text { number of wireless communication } \\
\text { interfaces }\end{array}$ & 4 \\
\hline mobility model & $\begin{array}{l}\text { MANET_Up_right } \\
\text { MANET_down_left }\end{array}$ \\
\hline Route active time (sec) & 3 \\
\hline Hello Interval $(\mathrm{sec})$ & uniform(1.1.1) \\
\hline Allowed hello loss & 2 \\
\hline Net surface $\left(\mathrm{km}^{2}\right)$ & 20 \\
\hline Node traversal time (sec) & 0.04 \\
\hline Route error rate limit (pkts/sec) & 10 \\
\hline Timeout buffer $(\mathrm{sec})$ & 2 \\
\hline AODV Local repair & Enable \\
\hline Time of simulation (hour) & 1 \\
\hline
\end{tabular}

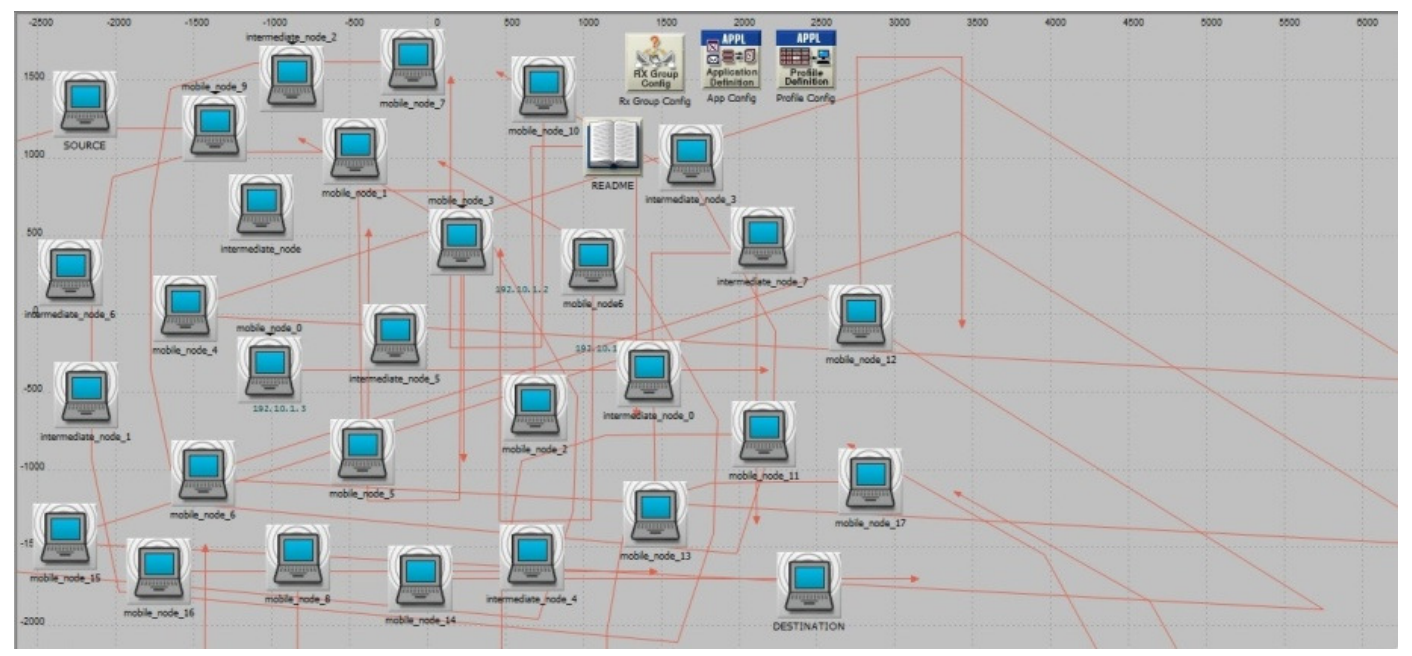

Figure 6. The Topology of a Thirty-Node MANET 


\subsection{The Results}

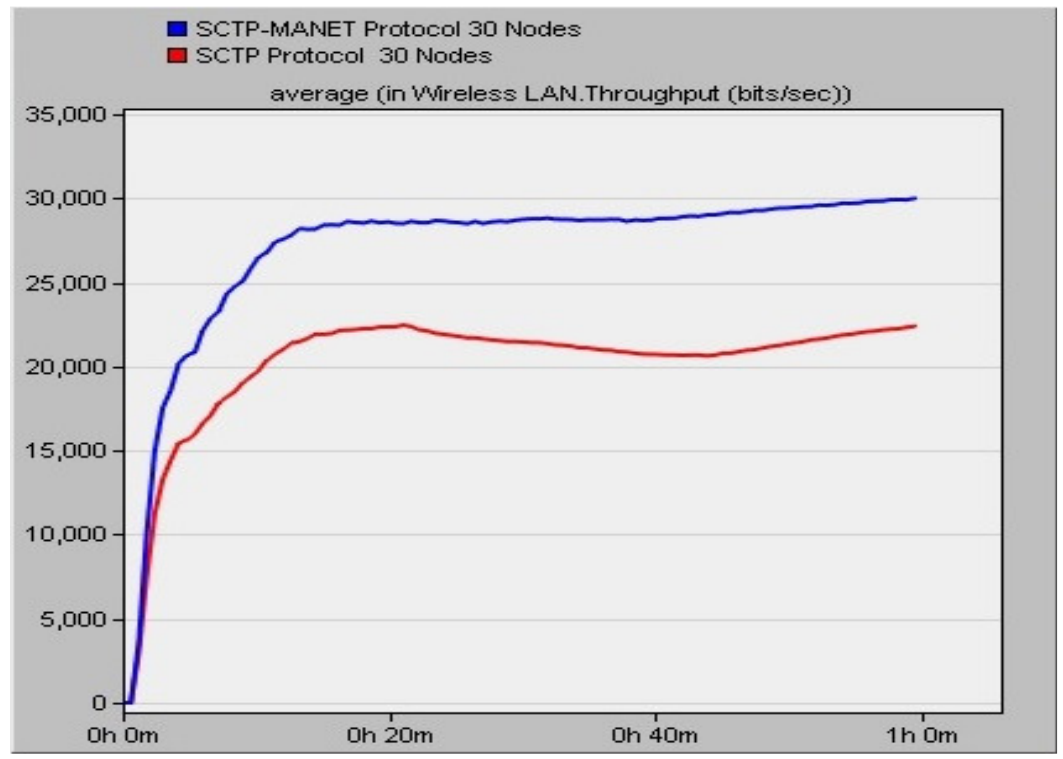

Figure 7. First scenarios thirty nodes MANET average throughput

Figure 7 demonstrates the average throughput of the first scenarios with (30 nodes). For these two (2) variants, we notice an important gap between the two (2) curves; because when SCTPMANET reaches a maximum value of $30000 \mathrm{bits} / \mathrm{sec}$, SCTP has a maximum value of 22000 bits/sec.

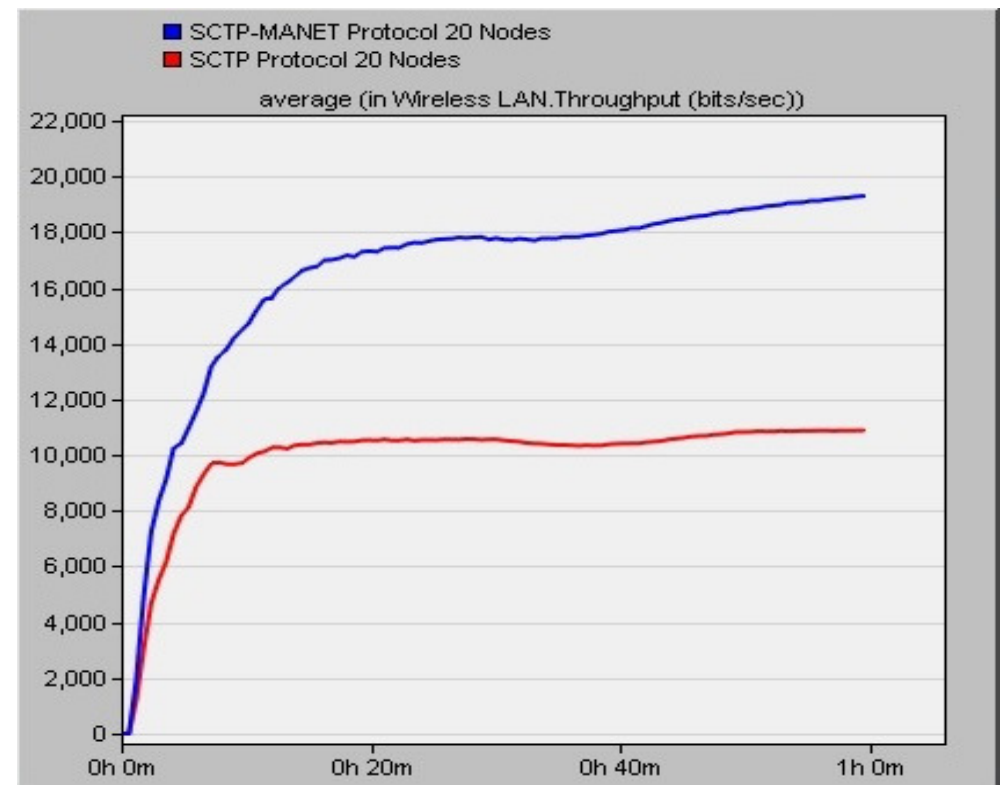

Figure 8. Second scenario twenty nodes MANET average throughput 
Figure 8 shows the average throughput of the second scenario (20 nodes). Compared to the first scenario, we notice a decrease of the throughput of the two variants. On the other hand, the gap between the average throughput of the two variants SCTP and SCTP-MANET rises.

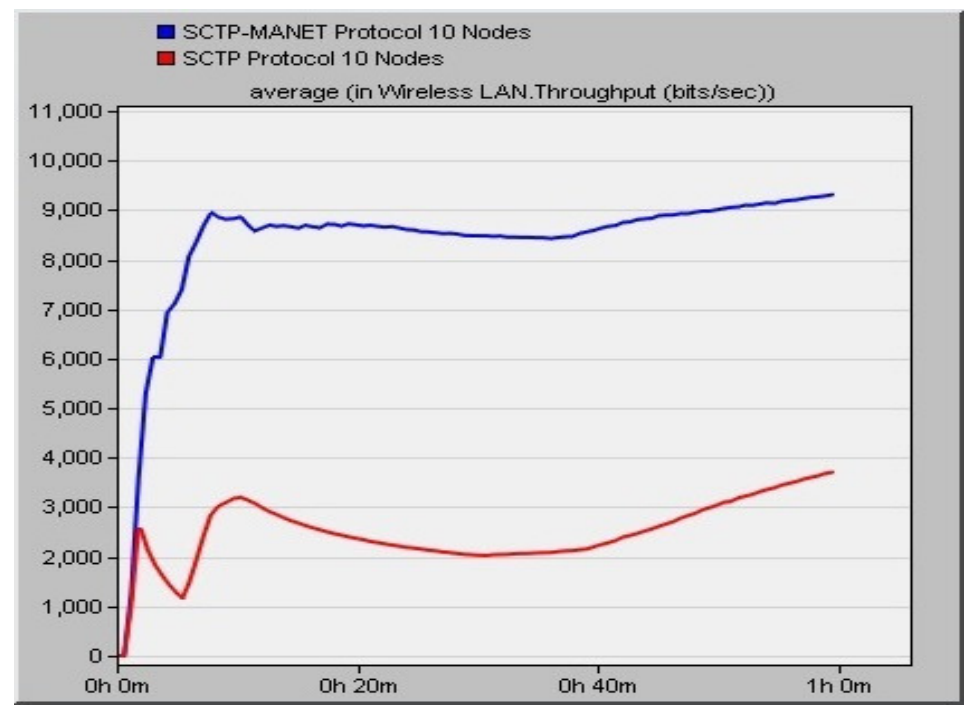

Figure 9. Third scenario ten nodes MANET average throughput

Finally, figure 9 illustrates the third scenario of a sparse network of only ten (10 nodes). The same could be said here: a decrease of the average throughput for the two variants of this scenario. Only an important gap grows between SCTP average throughput which reaches $3900 \mathrm{bits} / \mathrm{sec}$ and SCTP-MANET which has a maximum value of $9200 \mathrm{bits} / \mathrm{sec}$ that is almost three times higher.

\subsection{Results Discussion}

A comparative analysis of these three scenarios reveals that the decrease of the number of MANET nodes causes a fall in the average throughput. However, the use of a new extension SCTP-MANET shows even better average throughput compared to SCTP, with a gap that rapidly develops between these two variants (SCTP-MANET and SCTP) in sparse networks.

This could be attributed to the decrease of the number of the nodes participating in the routing, which involves a weak availability of the links. This directly results in recurrent transport path breakdowns. Compared to SCTP basic protocol, the extension SCTP-MANET manages better the recovery of the broken-down paths in sparse MANET; thanks to its anticipating method of detecting two alternative paths, as well as to its transparent switching from a broken down link to a functional one.

\section{CONCLUSION}

Compared to the standard SCTP version, the use of SCTP-MANET optimizes significantly the performances of sparse MANET which suggest weak link availability. The enhancement of such network performances is due to the fact that SCTP-MANET prevents transport path breakdowns. This extension works with a minimum of three active paths. In addition, the use of a direct 
transport and routing cross layer interface and specific SCTP-MANET messages suggests a better integration of Multihoming. The latter takes in charge realizing a transparent passage between the paths without data loss or network disconnection.

Finally, in order to enhance the contribution of this new extension to network performances, we suggest that future work in this area study the use of a similarity counter. Incremented when paths pass by the same nodes, this counter decides upon the worth of a secondary path.

\section{REFERENCES}

[1] C.S.R. Murthy, B. Manoj, Ad-hoc Wireless Networks: Architectures and Protocols, Prentice Hall, New Jersey, USA, 2004

[2] G. Angione, P. Bellavista, A. Corradi, E. Magistretti, A k-hop clustering protocol for dense mobile ad-hoc networks, in: International Conference on Distributed Computing Systems Workshops, vol. 01, 2006. doi:http://dx.doi.org/10.1109/ICDCSW.2006

[3] Marina M.K., Das S.R. “Ad hoc on-demand multipath distance vector routing”. WC\& MC, Vol. 6, Wireless Ad hoc Networks: Technology and Challenges,2006

[4] Thomas Kunz, "Implementation vs. simulation: Evaluating a MANET multicast protocol", in Proceedings of the Global Mobile Congress 2004, Shanghai, China, 129-134, 2004, Delson Group 2004

[5] A. Nilsson, C.E. Perkins, A.J. Touminen, R. Wakikawa, J.T. Malinen, AODV and IPv6 Internet access for ad hoc networks, Mobile Computer and Communications Review, 102-103, 2013.

[6] J. Yu, P. Chong, A survey of clustering schemes for mobile ad hoc networks, IEEE Commun. Surv. Tutor. 32-48, 2005

[7] Stewart R “Stream Control transmission Protocol” RFC 4960, IETF, Septembre 2007.

[8] R. Stewart, Q. Xie, M. Tuexen, S. Maruyama, M. Kozuka, Stream Control Transmission Protocol (SCTP) Dynamic Address Reconfiguration, RFC 5061, 2007.

[9] R. Stewart, Q. Xie, K. Morneault, C. Sharp, H. Schwarzbauer, T. Taylor, I. Rytina, M. Kalla, L. Zhang, V. Paxson, Stream Control Transmission Protocol, RFC 4960, 2007.

[10] Kelly, G.m. Muntean , P. Perry , J. Murphy, Delay-centric handover in SCTP over WLAN, Trans. Autom. Control Comput. Sci. 1-6, 2004.

[11] J.R. Iyengar, P.D. Amer, R. Stewart, Concurrent multipath transfer using SCTP multihoming over independent end-to-end paths, IEEE ACM Trans. Netw. 951-964, 2006.

[12] T. Stegel, J. Sterle, J. Bešter, A. Kos, SCTP association between multi-homedendpoints over NAT using NSLP, Electrotech. Rev, 277-284. 2008

[13] A. Nasipuri, R. Castaneda, S.R. Das, Performance of multipath routing for on-demand protocols in mobile ad hoc networks, Mobile Networks and Applications,339-349. 2001

[14] P. Goudarzi , Scalable video transmission over multi-hop wireless networks with enhanced quality of experience using Swarm in- telligence, Signal Process. Image Commun.

[15] Charoenpanyasak S.,Paillassa B.,"SCTP multihoming with Cross Layer Interface in Ad Hoc Multihomed Networks" IEEE Wi-Mob, New York USA, 2007.

[16] Kim K., "A Cross Layered Approach for Multihoming on SCTP in mobile Ad hoc Networks", TENCON (Taipei International Convention Center), IEEE Region 10 Conference, 2007.

[17] G. RAJKUMAR, DR. K. DURAISAMY, "A Review of Ad Hoc On-Demand Distance Vector Routing Protocol for Mobile Ad HocNetworks", in Journal of Theoretical and Applied Information Technology, Vol. 36 No.1, 2012

[18] R. Oliveira, M. Luis, L. Bernardo, R. Dinis, Towards reliable broadcast in ad hoc networks, IEEE Communications Letters 314-317. 2012

[19] Md. Nurul Islam, A. Kara, Throughput analysis of SCTP over a multi-homed association, Computer and Information Technology, CIT '06, in: The Sixth IEEE International Conference on Computer and Information Technology, Seoul, Korea, 110-110. 2006

[20] Introduction to OPNET Modeler. Networks Competence Area, COM. DTU, Retrieved from: [Online].Availableat:http://oldwww.com.dtu.dk/staff/vbi/EuroFGI/doc/EuroFGI_Intro2 Modeler.pdf. 
International Journal of Wireless \& Mobile Networks (IJWMN) Vol. 8, No. 5, October 2016

[21] Riverbed Technology, The Riverbed Splash Community, OPNET Modeler Space, the RSVP Refresh Reduction Extensions, 2013. <https://splash.riverbed.com/docs/DOC-3202>.

[22] Koltsidas G., Palvidou F., Kuladinithi K., Timm-Giel A., Gorg C., "Investigating the performance of multipath protocol for ad-hoc networks" The 1th Annual IEEE PIMRC, Septembre 2007. 